\title{
A GATE-based Monte Carlo Simulation of a Dual-layer Pixelized Gadolinium Oxyorthosilicate (GSO) Detector Performance and Response for Micro PET Scanner
}

\author{
Leonid L. Nkuba ${ }^{1,2}$, Innocent J. Lugendo ${ }^{1}$ and Idrissa S. Amour ${ }^{3}$ \\ ${ }^{I}$ Department of Physics, University of Dar es Salaam, P. O. Box 35063, Dar es Salaam, Tanzania. \\ ${ }^{2}$ Radiation Control Directorate, Tanzania Atomic Energy Commission, P. O. Box 743, Arusha, \\ Tanzania. \\ ${ }^{3}$ Department of Mathematics, University of Dar es Salaam, P. O. Box 35062, Dar es Salaam, \\ Tanzania. \\ *Corresponding author, e-mail: leonid_nkuba@yahoo.co.uk \\ Co-authors'e-mails: ilugendo26@gmail.com,idrissa.amour@gmail.com
}

Received 5 Jan 2021, Revised 10 Mar 2021, Accepted 21 Apr 2021, Published May 2021

DOI: https://dx.doi.org/10.4314/tjs.v47i2.9

\begin{abstract}
The purpose of this study was to simulate the GSO detector of a micro PET using GATE simulation platform. The performance and responses of the simulated GSO detector assembly were evaluated by comparing the simulated data to the experimental and XCOM data to validate the simulation platform and procedure. Based on NEMA NU-4 2008 protocols, the performance of GSO detector in terms of sensitivity was simulated and compared to the experimental data. Similarly, the GSO detector response to photons interaction was simulated and compared against the XCOM data for absorbed intensity ratio in the GSO detector and survived intensity ratio in $\mathrm{Pb}$ blocks. Results showed that simulated and experimental sensitivities agreed well with $R^{2}$ of 0.995 and two overlapping bands at $95 \%$ confidence. An agreement with $R^{2}$ of 0.972 and 0.973 as well as with overlapping bands at $95 \%$ confidence was obtained in simulated and XCOM data for absorbed and survived intensity ratio in the GSO detector and $\mathrm{Pb}$ blocks, respectively. The observed agreements demonstrate the accuracy of the simulation method to mimic the behaviour of the GSO detector. The validated GATE algorithm for micro PET scanner is therefore recommended for simulation and optimisation of collimator design in further studies.
\end{abstract}

Keywords: GATE simulation, Experimental data, XCOM data, GSO detector, micro PET.

\section{Introduction}

Small animal Positron Emission Tomography (PET), also called micro PET, is a preclinical molecular imaging tool that allows measurement of the spatial distribution of radiotracers, which map physiological and metabolic functions in small animals such as mice or rats. A key component of PET imaging systems is the detector element, an area with a long and rich history. Early PET detector assemblies were based on the use of sodium iodide scintillators activated with thallium [NaI(Tl)] coupled to photomultiplier tubes (PMTs) (Lewellen 2008, Khalil 2017). Although various technological alternatives have emerged, for instance, wire chambers and solid-state devices (Park et al. 2007a, Park et al. 2007b, Lewellen 2008), the scintillatorbased technology remains the most common primary detector crystal for PET systems. Ideally, a scintillator material for micro PET detector should have fast decay time, high light 507 
output, high density as well as high atomic number and must be cheap to produce and easily machinable so that fine segmentation for high spatial resolution can be achieved (Lewellen 2008, Khalil 2017).

The $\mathrm{Be}_{4} \mathrm{Ge}_{3} \mathrm{O}_{12}$ (Bismuth Germanate-BGO) scintillator has been considered the state-ofthe-art detector crystal for PET systems (Vandenbroucke et al. 2010, Nikolopoulos et al. 2014) and replaced $\mathrm{NaI}(\mathrm{Tl})$ detectors in early PET prototypes (Khalil 2017). However, due to their high detection efficiencies, the $\mathrm{LuSiO}_{5}$ (Lutetium Oxyorthosilicate-LSO) and $\mathrm{Gd}_{2} \mathrm{SiO}_{5}$ (GSO) scintillators have become the best competitors of the BGO (Nikolopoulos et al. 2014). The GSO doped with cerium (Ce:GSO) possesses excellent properties, including high light yield, fast decay time, and good radiation hardness (Tanaka et al. 1998, Qi et al. 2003, Lee et al. 2008). The light yield of $\mathrm{Ce}: \mathrm{GSO}$ is twice as high, and the decay time is $1 / 5$ (fast decay times) of that of commonly used BGO. The GSO crystal has been found with an additional interest in parallax error correction by using dual layer of GSO or coupling it with other crystals such as LSO (Khalil 2017). Moreover, irradiation hardness (radiation resistance) of the GSO crystal is prominent (Tanaka et al. 1998), and was reported in the order of $>10^{6}$ Gy compared to 10 Gy reported for $\mathrm{BGO}$ and $\mathrm{NaI}(\mathrm{Tl})$ scintillators for gamma rays, hence, making it widely useful in various applications in nuclear physics, nuclear medical imaging (e.g., PET detectors) and high-energy physics (Matulewicz et al. 1996, Kawade et al. 2011). Nevertheless, other scintillator materials, such as $\mathrm{LuAlO}_{3}$ (Lutetium Aluminium PerovskiteLuAP) and $\mathrm{YAlO}_{3}$ (Yttrium Aluminium Perovskite-YAP) have been used as well (Nikolopoulos et al. 2014, Okumura et al. 2015).

The superior properties of the GSO detector have led to several efforts of developing PET imaging system using Ce:GSO as their detection units. A study by Okumura et al. (2015) developed a non-commercial micro PET prototype that employs a dual-layer
$\mathrm{Gd}_{2} \mathrm{SiO}_{5}$ (GSO) cerium (Ce) doped detector crystals. Among the important aspects of the developed prototype are the overall performance and response of the detector system. Therefore, a need to study the performance and responses of the GSO detector for use in a micro PET system has risen. One of the sound methods for studying the performance and responses of the detection system in such a situation is the use of Monte Carlo simulations (Jan et al. 2005, Lamare et al. 2006). Several Monte Carlo simulation platforms can be used for modelling the detection system and other parts of the imaging system in nuclear medicine (Buvat and Castiglioni 2002).

In this study, Geant4 Application for Tomographic Emission (GATE) simulation toolkit, which encapsulates GEometry ANd Tracking (GEANT4) libraries was used. GATE was designed for PET and Single Photon Emission Tomography (SPECT) to simulate various imaging parameters including the collimators and other components located behind the detector crystal, which are normally assumed in other simulation platforms (Santin et al. 2003, Pietrzyk 2017). However, the process of developing a simulation algorithm for studying the performance and responses of detector system is not a straightforward task. The developed algorithm must first be validated using existing and or known data and parameters. Therefore, this study sets out to simulate and investigate the performance and responses of the GSO detector for the micro PET system using GATE Monte Carlo simulation. Finally, the simulated data were compared to the experimental and $\mathrm{XCOM}$ data to validate the simulation platform and procedure.

\section{Materials and Methods GATE simulation platform}

To simulate the GSO detector model for the micro PET system, GATE version 9.0 was used. This code is based on well-validated GEANT4 (geant4-10-06-patch-02) libraries and dedicated to nuclear imaging (PET and 
SPECT) applications, computed tomography (CT), optical imaging (bioluminescence and fluorescence) and radiotherapy (Santin et al. 2003, Pietrzyk 2017). GEANT4 was developed at the European Organization for Nuclear Research (CERN) to simulate the passage and behaviour of particles (e.g. electrons, protons, neutrons and photons) through matter (Allison et al. 2016). The design and architecture of GATE has been described in detail (Santin et al. 2003, Pietrzyk 2017). On top of GEANT4, GATE simulation platform includes specific modules developed to meet specific requirements encountered in PET. In the GATE, a user-friendly mechanism based on scripts (or commands) is used to define all the simulation parameters including world (hypothetical volume), scanner geometry, detector, phantom, source distribution, geometry and modelling (Pietrzyk 2017).

\section{Description of the prototype scanner}

The prototype is composed of a micro PET system, which consists of pixelized GSO scintillation detector modules (Figure 1) developed by Okumura et al. (2015). The GSO scintillation detector modules coupled with Flat Panel Photomultiplier Tubes [(FPPMT: Hamamatsu photonics H8500, $8 \times 8$ anodes) sometimes called Position-Sensitive Photomultiplier Tubes (PSPMTs) (Yamamoto et al. 2012)] at an angle of $22.5^{\circ}$ were arranged in a hexagonal shape to form a ring-like micro PET detector system. Each detector module consists of a dual layered GSO block; hence the prototype had sixteen GSO modules in a ring (Figure 1). The inner and outer diameters of the ring are $95 \mathrm{~mm}$ (radius $47.5 \mathrm{~mm}$ ) and $130 \mathrm{~mm}$ (radius $65 \mathrm{~mm}$ ), while the axial field of view (FOV) is $37.5 \mathrm{~mm}$. The dual layers of a single GSO block are made of GSO scintillators doped with cerium (Ce) concentration of $1.5 \mathrm{~mol} \%$ (fast decay time: 39 $\mathrm{ns}$ ) and $0.4 \mathrm{~mol} \%$ (slow decay time: $69 \mathrm{~ns}$ ) for the first or lower layer and second or upper layer, respectively. These two layers of GSO scintillators in a detector module were optically coupled to determine the depth of interaction from the differences in decay times.

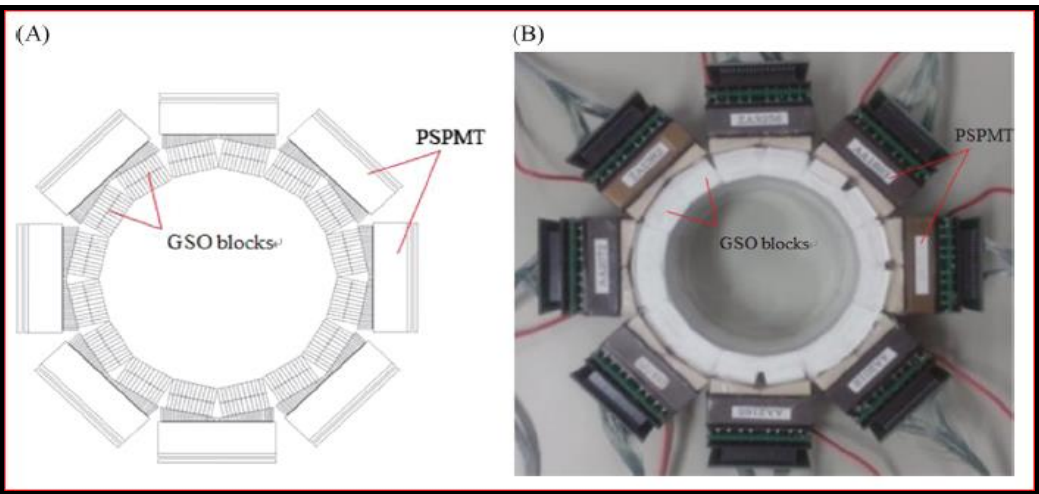

Figure 1: A schematic diagram of the prototype composed of a micro PET detection system: (A) detector ring, and (B) a photo of developed hexagonal-shaped detector ring (Okumura et al. 2015).

As shown in Figure 2, the GSO pixels are arranged in a $11 \times 15$ matrix with a $0.1-\mathrm{mm}$ thick $\mathrm{BaSO}_{4}$ reflector at a pitch of 1.7 or 2.5 $\mathrm{mm}$. The sizes of these GSO cells or pixel crystals were $1.6 \times 2.4 \times 7 \mathrm{~mm}^{3}$ and $1.6 \times 2.4 \times$ $8 \mathrm{~mm}^{3}$ for $1.5 \mathrm{~mol} \% \mathrm{Ce}$ and $0.4 \mathrm{~mol} \% \mathrm{Ce}$, respectively. Each detector block has a surface area of $18.7 \mathrm{~mm} \times 37.5 \mathrm{~mm}\left(701.25 \mathrm{~mm}^{2}\right)$ with $15 \mathrm{~mm}$ thickness $(8 \mathrm{~mm}$ in the first or lower layer and $7 \mathrm{~mm}$ in the second or upper layer). Other details regarding the detector unit of this micro PET prototype were described by Okumura et al. (2015). 
Nkuba et al. - A GATE-based Monte Carlo simulation of a dual-layer pixelized gadolinium ...

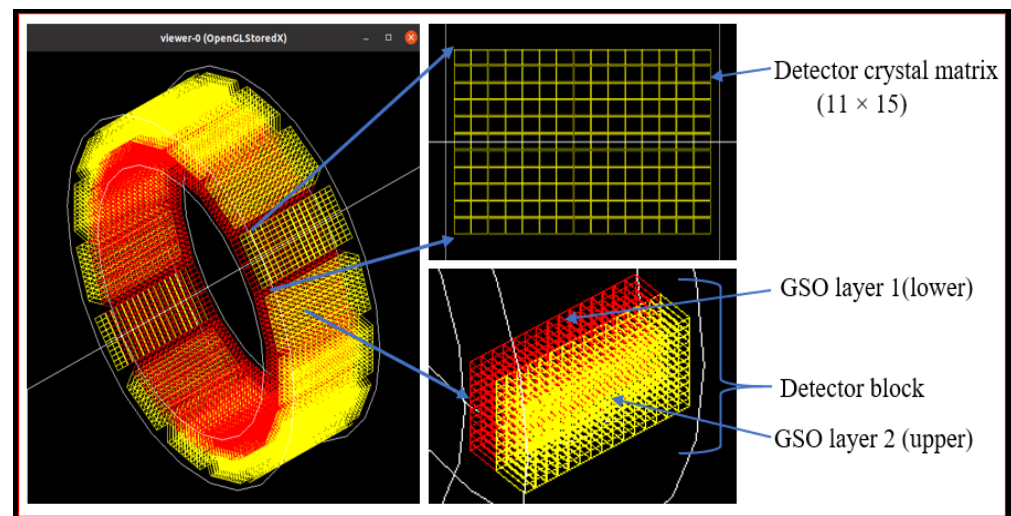

Figure 2: The simulated GSO detector geometry of the micro PET scanner. The detector pixel matrix and crystal layers are shown.

\section{GATE Monte Carlo model of the scanner}

To simulate the detector of a micro PET scanner, the scanner geometry, the pixelated GSO crystal (dual layer and crystal array), phantom, physical process and the radioactive source should be modelled. The scanner geometry and its components were modelled in the world volume. The world volume is a box centred at the origin. It can be of any size but has to be large enough to include the entire scanner simulation geometry. The tracking of any particle stops when it escapes from the world volume. To model detection system, the detector crystals were defined and attached as sensitive volumes. GATE simulation only stores hits for those volumes attached to a sensitive detector. Hits regarding interactions occurring in non-sensitive volumes are lost. A volume must belong to a system before it can be attached to a sensitive detector. Hits, occurring in a volume, cannot be scored in an output file if the said volume is not connected to a system and unattached to a sensitive detector (Pietrzyk 2017).

Phantoms are geometry to be scanned; they are modelled and defined as sensitive volume to track physical processes of the full gamma rays from the vertex point to the end. For validation processes, a cubic acrylic phantom $\left(10 \times 10 \times 10 \mathrm{~mm}^{3}\right)$ was modelled on the basis of NEMA NU-4 2008 protocols for sensitivity analysis (Riehakainen 2018). The physical processes occurring in the phantom include Compton scattering, Rayleigh scattering and Photoelectric interactions (Pietrzyk 2017), which yield information about the photon. This information is necessary to determine whether a photon reaching the detector has been scattered or not.

Physical processes include all possible interaction mechanisms of the incident radiation from the source that occur in the phantom, lead $(\mathrm{Pb})$ block (used in validation processes) and detector crystals (Pietrzyk 2017). In our simulation, we used the standard model (1 $\mathrm{keV}$ and $100 \mathrm{TeV})$ for the photoelectric effect, Compton scattering, electron ionization and Bremsstrahlung, while Penelope model ( $250 \mathrm{eV}$ and $1 \mathrm{GeV}$ ) was used for Rayleigh scattering (Pietrzyk 2017). For the annihilation, an improvement has been developed in GATE simulation tool kit to consider the gamma-gamma non-collinearity, and therefore, positron-electron annihilation does not need a physical process model selection (Pietrzyk 2017). After setting the physics description, the GATE user must initialize the simulation with the following command: /gate/run/initialize. This process triggers the calculation of the cross-section tables, and the boundaries are checked as well.

In GATE simulation, the digitizer preprocesses the hits by sorting, summing (adder) and regrouping (readout) them to create 
singles. The singles are time-stamped and stored in the event's history. In our simulation, the coincidences are sorted out as a function of the time coincidence window, which was set to $20 \mathrm{~ns}$, with $500 \mathrm{~ns}$ as the offset value. In the blurring module, the GSO detector parameters used are intrinsic energy resolution of 0.099 (9.9\%) for $511 \mathrm{keV}$ and $0.284(28.4 \%)$ for 372 $\mathrm{keV}$ for simulation of sensitivity and detector response, respectively. The time or temporal resolution (FWHM) of GSO is $0.549 \mathrm{~ns}$.
Other parameters include energy threshold of $350 \mathrm{keV}$ and uphold of $1000 \mathrm{keV}$ as well as energy threshold of $350 \mathrm{keV}$ and uphold of 750 $\mathrm{keV}$ for simulation of detector response and sensitivity, respectively. Lastly, coincidence sorter module (picks out those singles, which are in coincidence) was included in the digitizer level in our simulation for sensitivity (Figure 3).

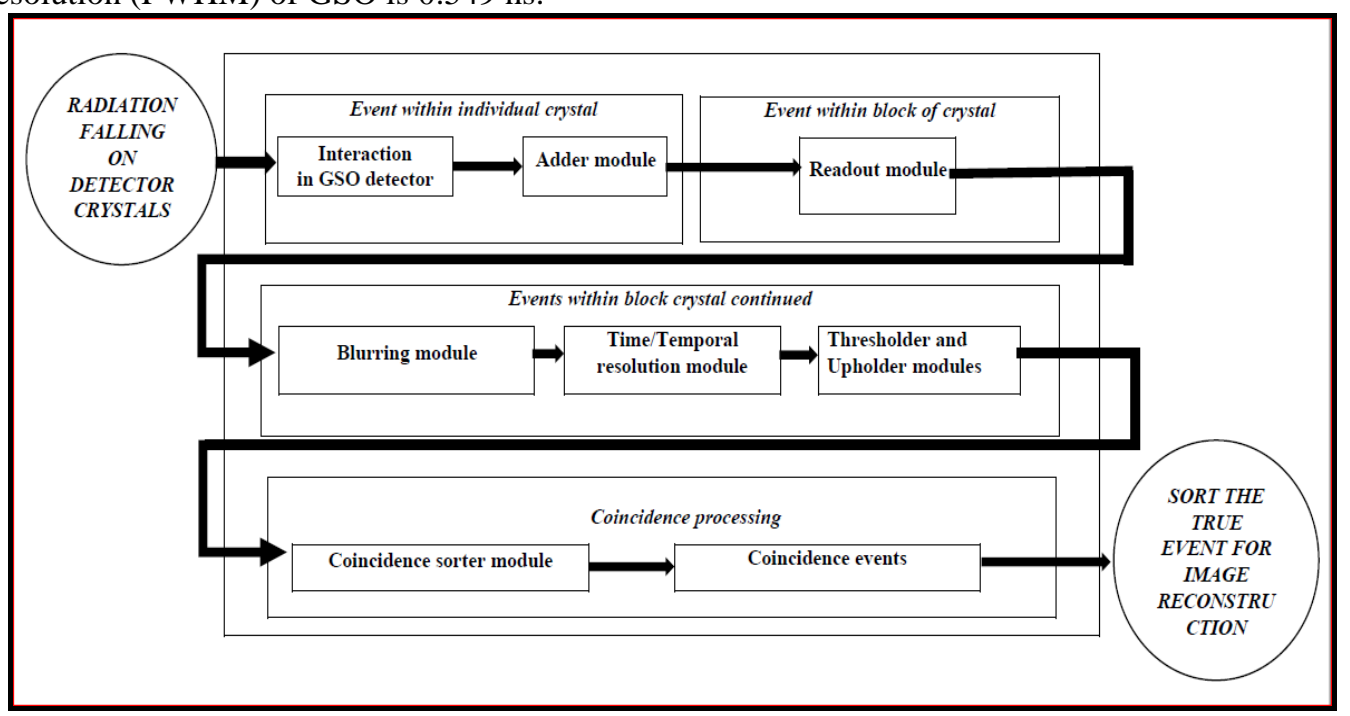

Figure 3: Schematic diagram of the digitizer chain.

\section{Source description, output setup and time management}

The definition of radioactive sources includes the particles emitted (e.g., ion, gamma, positrons), position and geometry (volume), the direction of emission (solid angle), energy (spectrum) and activity ( $\mathrm{Bq}$ ). The activity determines the decay rate of the source during the simulation acquisition time. In this study, Pencil Beam Sources (PBS) with energies of $372 \mathrm{keV}, 414 \mathrm{keV}, 511 \mathrm{keV}, 617$ $\mathrm{keV}, 695 \mathrm{keV}, 869 \mathrm{keV}$ and $1000 \mathrm{keV}$ were used for assessing detector responses to photon interactions. The $1000 \mathrm{keV}$ PBS was used for ratio determination. Based on NEMA NU-4 2008 protocols, we modelled the ${ }^{22} \mathrm{Na}$ source with $400 \mathrm{kBq}$ activity for sensitivity determination.
The GATE simulation output was stored in the ROOT file format (Brun and Rademakers 1997). In which, the ROOT output consists of several folders, which include two hit folders and one coincidence folder. Hits contain information about the energy deposition of a step, and the position and time of a step. Numbers of hits were used in determining detector responses based on absorbed or surviving intensity ratio. In a coincidence folder, which is of interest in sensitivity determination, the event ID number and the number of Compton and Rayleigh interactions that have occurred during the tracking of each photon are stored and used in the classification or analysis of true, random and scattered coincidences (Pietrzyk 2017). 
Before the time management, a random number generator engine and its seed are defined. After that, the commands for time management were written. Time management defines the beginning and end of the acquisition process, this step is defined also along with the step time (time slice), which finally indicates the number of runs for a particular simulation. In our simulation, for absorbed and survived intensity ratio in GSO detector and cuboid lead $(\mathrm{Pb})$ blocks, we set time start $=0$, time end $=100 \mathrm{~s}$, and time slice $=1 \mathrm{~s}$. For sensitivity analysis, time end was set to $60 \mathrm{~s}$. In a simulation, each slice corresponds to a particular run.

\section{Validation of the GATE simulation algorithm for GSO detector of a micro PET Sensitivity}

The sensitivity of a scanner represents the efficiency to detect the coincident annihilation radiation. In this developed simulation model, sensitivity is defined as counts (number of true events) per second per Becquerel (cps/Bq) and was assessed based on NEMA NU-4 2008 protocols. The sensitivity profile of the micro PET was measured using a calibrated ${ }^{22} \mathrm{Na}$ point source, with the activity of $400 \mathrm{kBq}$ enclosed in $10 \times 10 \times 10 \mathrm{~mm}^{3}$ acrylic (polymethyl methacrylate - PMMA) cubic phantom. Phantom was placed in nine axial ( $\mathrm{z}$ axis) locations at $5 \mathrm{~mm}$ steps and the count rate was acquired for $60 \mathrm{~s}$ for each location set. The time coincidence window was fixed at $20 \mathrm{~ns}$, while the lower and upper energy windows were fixed at $350 \mathrm{keV}$ and $750 \mathrm{keV}$, respectively. The number of true, random, and scattered coincidences from experimental data was acquired for sensitivity determination. According to NEMA NU-04 2008 protocols, the experimental sensitivity $\left(S_{i}\right)$ in $\mathrm{cps} / \mathrm{MBq}$ was calculated using Equation 1 (Riehakainen 2018).

$$
S_{i}=\frac{R_{T i}-R_{B i}}{A_{S}}
$$

where $R_{T i}$ and $R_{B i}$ are true count rate (true counts per $60 \mathrm{~s}$ ) and Background count rate (Background counts per $60 \mathrm{~s}$ ). $A_{s}$ is the source activity (in this study, $400 \mathrm{kBq}$ ).

In sensitivity simulation, a $0.25 \mathrm{~mm}$ diameter ${ }^{22} \mathrm{Na}(400 \mathrm{kBq})$ point source in an acrylic (PMMA) cubic phantom was modelled as shown in Figure 4. The experimental procedures for sensitivity were adhered to the simulation. To obtain the number true, random and scattered coincidence events from simulated data, a C++ sorter program was written. Since in simulation we did not account for the background count rate, the simulated sensitivity $\left(S_{i}\right)$ was calculated using Equation 1 after equating the term $R_{B i}$ to zero.

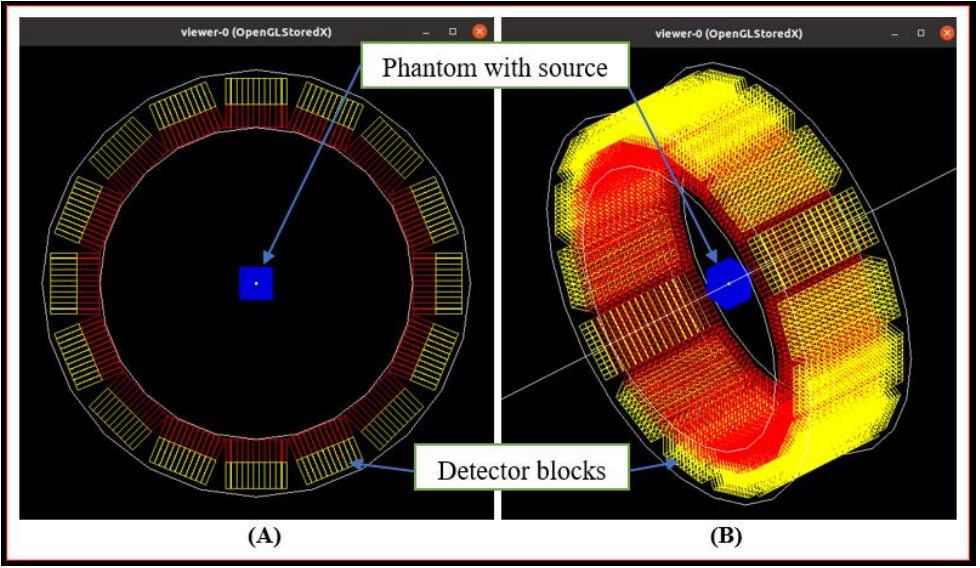

Figure 4: Simulated micro PET geometry for sensitivity determination: (A) front view of the detector geometry, and (B) side view $\left(60^{\circ}\right)$ of the detector geometry. 
The absolute sensitivities $\left(S_{a}\right)$, given in percentage $(\%)$ for experimental and simulated data were determined using Equation 2 by correcting the sensitivities $\left(S_{i}\right)$ given in $\mathrm{cps} / \mathrm{MBq}$ for the branching ratio of the source, which is 0.906 for ${ }^{22} \mathrm{Na}$ (Riehakainen 2018).

$$
S_{a}=\frac{S_{i}}{0.906} \times 100 \%
$$

where $S_{a}$ and $S_{i}$ represents sensitivity (cps/MBq) and absolute sensitivity (\%) respectively.

The agreement between the simulated and experimental absolute sensitivities of micro PET scanner was assessed using the coefficient of determination $\left(R^{2}\right)$ and Gaussian fit model with $95 \%$ confidence bands. The $R^{2}$ usually shows if the model fits well the data (the goodness of fit), a higher coefficient is an indicator of better goodness of fit for the observation. Note that, as the value of $R^{2}$ is getting closer to one, the closer the simulated and experimental measured values are.

\section{Absorbed intensity ratio in $\mathrm{Gd}_{2} \mathrm{SiO}_{5}$ (GSO) detector crystals}

The XCOM online software gives us the mass attenuation coefficients $\left(\mu_{m}\right)$ for energies $372 \mathrm{keV}, 414 \mathrm{keV}, 511 \mathrm{keV}, 617 \mathrm{keV}, 695$ $\mathrm{keV}, 869 \mathrm{keV}$ and $1000 \mathrm{keV}$ for the GSO scintillating material (XCOM website). The total length $(x)$ of GSO scintillator material in layer1 and layer2 is $1.5 \mathrm{~cm}$ (Yamamoto et al. 2015, Fukuchi et al. 2017). The density ( $\rho)$ of the scintillating material is $6.7 \mathrm{~g} \mathrm{~cm}^{-3}$ (Pietrzyk 2017). Therefore, the linear attenuation coefficient $(\mu)$ can be determined by multiplying the mass attenuation coefficient $\left(\mu_{m}\right)$ with the density of the material $(\rho)$. Next, through multiplying the linear attenuation coefficient with the length of the scintillating material, and then substitute $(\mu x)$ in Equation 3 below we obtain the fraction of gamma rays that survived after traversing the crystal length.

$$
I=I_{0} e^{-\mu x}
$$

Subtracting the fraction value from unity gives the number of absorbed gamma rays in the detector crystal. To obtain the absorbed intensity ratio value, a ratio of the number obtained after subtraction for particular energy (e.g. $372 \mathrm{keV}$ ) is taken with the subtracted value obtained for the $1000 \mathrm{keV}$ gamma ray.

In GATE simulations, a Pencil Beam Source (PBS) was used, where by seven runs were acquired each at $100 \mathrm{~s}$ using different pencil beams. Each beam had $10^{5}$ number primaries. In each simulation, a photon beam interacts directly with the $\mathrm{Gd}_{2} \mathrm{SiO}_{5}$ (GSO) scintillation detector crystals (Figure 5), then the total Hits (from the root output) were noted for each run. Finally, a ratio of these numbers obtained is taken with the value obtained for the $1000 \mathrm{keV}$ gamma ray to produce the absorbed intensity ratio in GSO detector crystals.

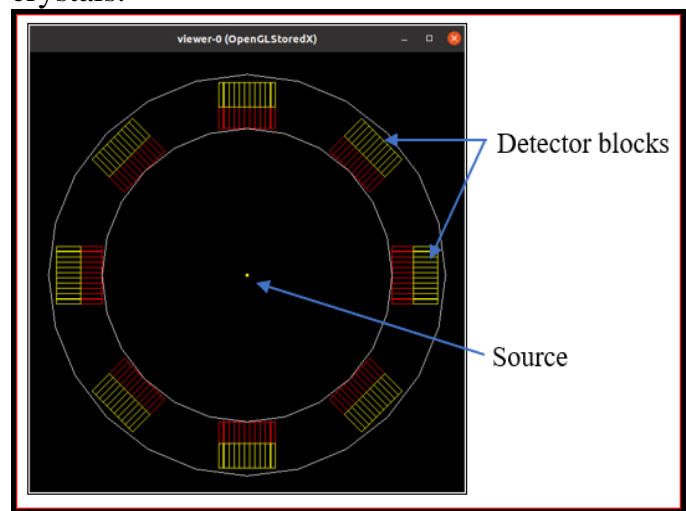

Figure 5: GATE-simulated ring geometry of $\mathrm{Gd}_{2} \mathrm{SiO}_{5}$ detectors and source at the centre.

\section{Survived intensity ratio after interacting with cuboid lead (Pb) blocks}

The mass attenuation coefficients $\left(\mu_{m}\right)$ for $372 \mathrm{keV}, 414 \mathrm{keV}, 511 \mathrm{keV}, 617 \mathrm{keV}, 695$ $\mathrm{keV}, 869 \mathrm{keV}$ and $1000 \mathrm{keV}$ energies for lead $\mathrm{Pb})$ material were obtained from the XCOM online software (XCOM website). The penetration depth $(x)$ in the lead block is $2.5 \mathrm{~cm}$ and the density $(\rho)$ of lead is $11.34 \mathrm{~g} \mathrm{~cm}^{-3}$ 
(Pietrzyk 2017). A similar procedure as given above for GSO detector crystal was followed to calculate the survived intensity. But here, we do not subtract the quantity from unity since we only require the survived intensity.

In GATE simulations, eight-cuboid lead block (in blue, as shown in Figure 6) each with $25 \mathrm{~mm} \times 18.7 \mathrm{~mm} \times 37.5 \mathrm{~mm}$ were attached in front of the micro PET's GSO detector modules. Then, seven runs were acquired each at $100 \mathrm{~s}$ using different pencil beams, each beam has $10^{5}$ number primaries. The generated photon beam interacts first with lead blocks, the survived photon beams after traversing the lead blocks finally interact directly with the $\mathrm{Gd}_{2} \mathrm{SiO}_{5}$ (GSO) detector crystals to produce hits. The total produced Hits (from the root output) were noted for seven runs.

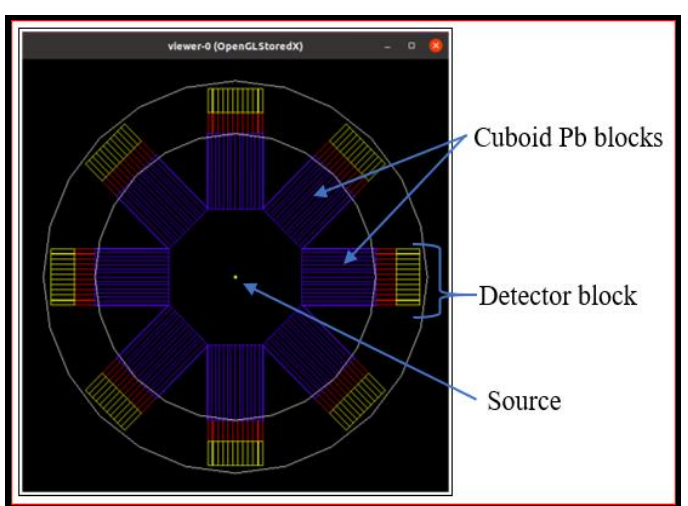

Figure 6: GATE-simulated ring geometry of GSO detectors with lead $(\mathrm{Pb})$ blocks in front.

Finally, a ratio of these numbers obtained is taken with the value obtained for the $1000 \mathrm{keV}$ gamma ray to generate the survived intensity ratio. The coefficient of determination $\left(R^{2}\right)$ and polynomial data fit model with $95 \%$ confidence band were used to assess the agreement between the GATE simulated and XCOM data.

\section{Results and Discussion Sensitivity}

The GSO detector sensitivities obtained from the simulation and experimental measurements are presented in this section. The obtained experimental absolute sensitivities (\%) resemble to those presented in Yamamoto et al. (2015) for the same micro PET scanner. The absolute sensitivity profile along the axial direction is plotted in Figure 7A, which shows GATE simulated and experimental peak absolute sensitivities of $1.71 \%$ and $1.70 \%$, respectively. The peak absolute sensitivities were acquired at the centre of the field of view. The relative error of less than $10 \%$ was observed for peak sensitivities. The coefficient of determination $\left(R^{2}\right)$ for the simulated and experimental data for absolute sensitivity was $0.995\left(R^{2}\right.$ is closer to 1). Figure 7B shows a Gaussian fitted model, which produced two overlapping bands at $95 \%$ confidence. The overlapping confidence bands indicate that some of the variance in the simulated data can be explained by experimental data.

Therefore, with the coefficient of determination $\left(R^{2}\right)$ of 0.995 and two overlapping confidence bands, there is an agreement between the simulated and experimental absolute sensitivities. Hence, the observed agreements suggest that the current GATE simulation algorithm is capable of modelling the absolute sensitivity of the micro PET scanner under the study. 


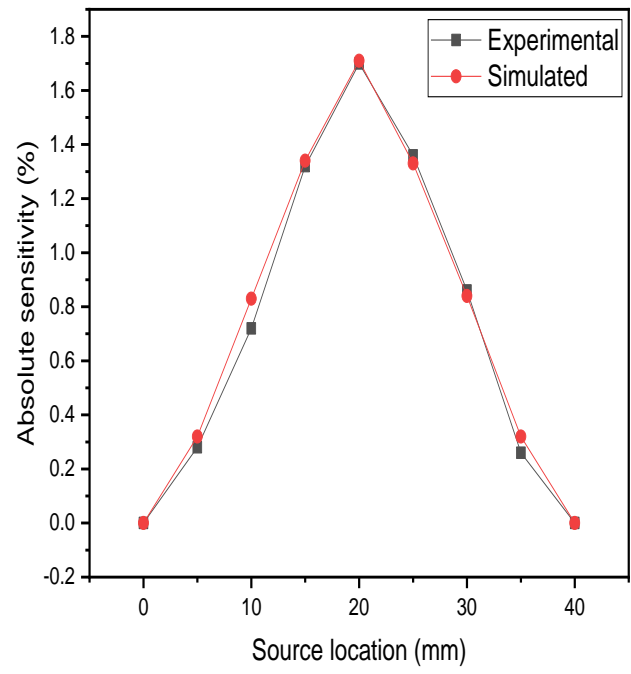

(A)

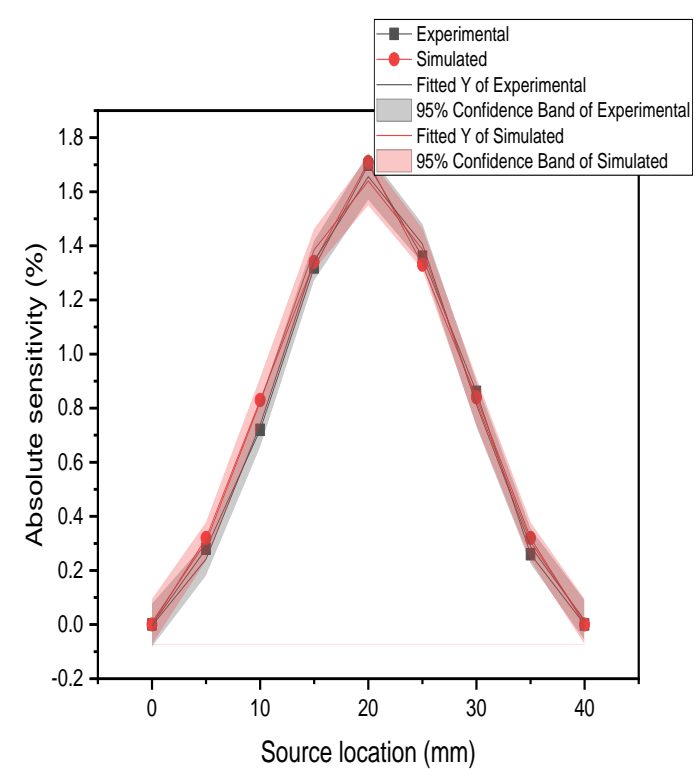

(B)

Figure 7: Experimental and simulated sensitivity profiles of a GSO detector for a micro PET: (A) Sensitivity data point plot and (B) Gaussian fitted sensitivity data points with $95 \%$ confidence band.

The observed discrepancies between the simulated and experimental data are due to the limitations of the photomultiplier tubes (PMTs) resolution and the absence of modelling of light shielding between the detector blocks (Toufique et al. 2013). Simulations without crystal reflector (light shielding between detector block) usually overestimate the simulated sensitivity by $10 \%-12 \%$ compared with experimental sensitivity (Jan et al. 2005) and this overestimation was evidenced by Schmidtlein et al. (2006) and Jakoby et al. (2009). In addition, the scanning bed was not taken into account, hence less scatter was observed in GATE-simulated data. Furthermore, the application of a varied quantum efficiency factor (QE) in the digitization process, might provide better agreement between simulated and measured sensitivities (Schmidtlein et al. 2006, Saaidi et al. 2017).

\section{Absorbed intensity ratio in $\mathrm{Gd}_{2} \mathrm{SiO}_{5}(\mathrm{GSO})$ detector crystals}

The absorbed intensity ratios obtained using GATE simulation and those predicted using the online XCOM software are plotted in Figure 8A. The coefficient of determination $\left(R^{2}\right)$ for the simulated and XCOM predicted data for absorbed intensity ratios in GSO detector crystal was 0.972 . As the value of $R^{2}$ approaches 1 (97.2\%), the closely GATE simulated and XCOM results are, hence this observation prove that there is an agreement between GATE simulated and XCOM measured values for absorbed intensity ratio in GSO crystals. 


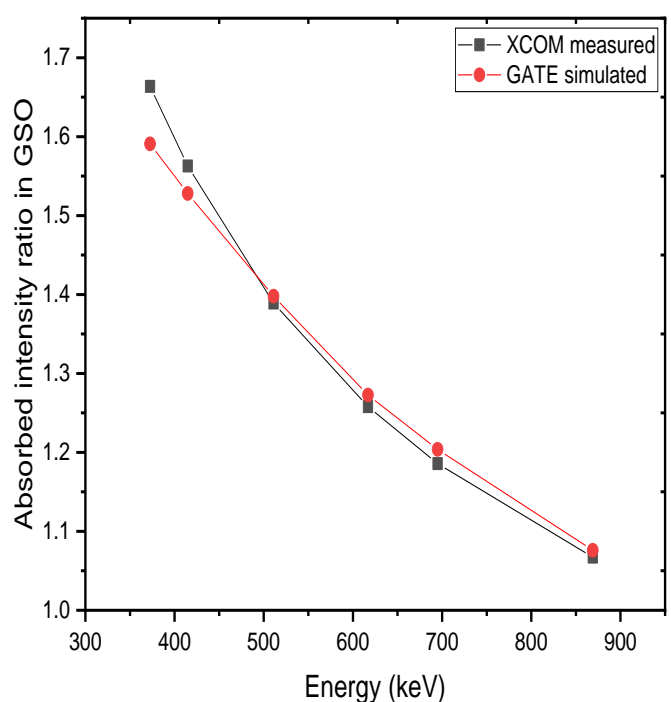

(A)

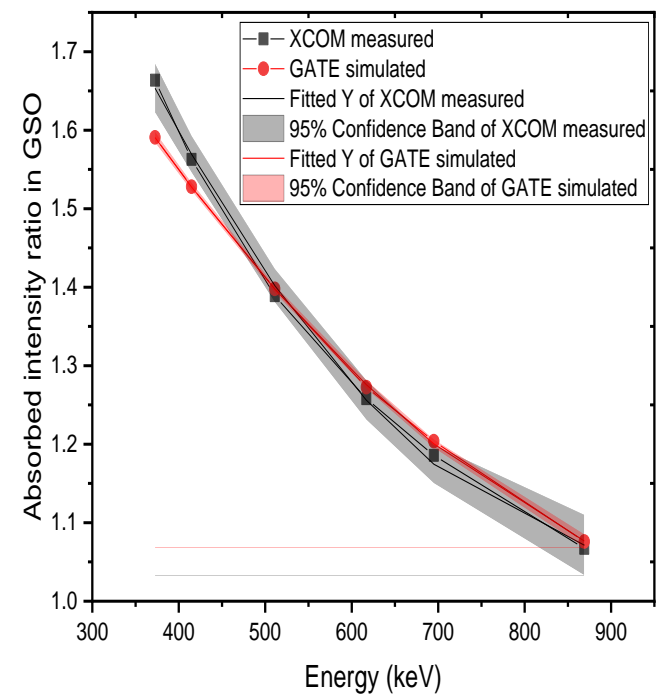

(B)

Figure 8: $\mathrm{XCOM}$ and GATE simulated data for absorbed intensity ratio in GSO detector: (A) shows data points while (B) shows the polynomial-fitted data.

As shown in Figure 8B, the polynomial fit model produced two overlapping bands at $95 \%$ confidence. Also, the overlapping confidence bands indicate that some of the variances in the simulated data can be explained by experimental data. Therefore, the observed agreements show that GATE simulation platform is capable of mimicking the GSO detector responses of the micro PET prototype under the study. The observed minor differences between GATE simulated and XCOM measured data for absorbed intensity ratio in GSO could be mainly due to photon scattering and its subsequent detection by the same crystal, but in a different module during simulations.

\section{Survived intensity ratio after interacting with cuboid lead (Pb) blocks}

The survived intensity ratios obtained using GATE and those predicted using the online XCOM software are plotted in Figure 9A. The results show that there is an agreement between the GATE simulated and XCOM data for survived intensity ratio in a cuboid lead $(\mathrm{Pb})$ block-placed in front of the GSO detector crystals because the coefficients of determination $\left(R^{2}\right)$ for the two data set is 0.973 and the polynomial-fitted data produced two overlapping bands at $95 \%$ confidence (Figure 9B). Since the value of $R^{2}$ is closer to 1 $(97.3 \%)$, this implies that the simulated and experimental measured values are also closer.

Also, by having two overlapping bands at 95\% confidence (Figure 9B) implies that some variances in the simulated data can be explained by XCOM data. This agreement demonstrates the reliability of GATE for accurately predicting GSO detector responses of the micro PET scanner. The observed discrepancies could be due mainly to photon scattering (in detector crystals) and its subsequent detection by the same crystal, but in a different module that resulting to the increasing number of hits in simulations. 


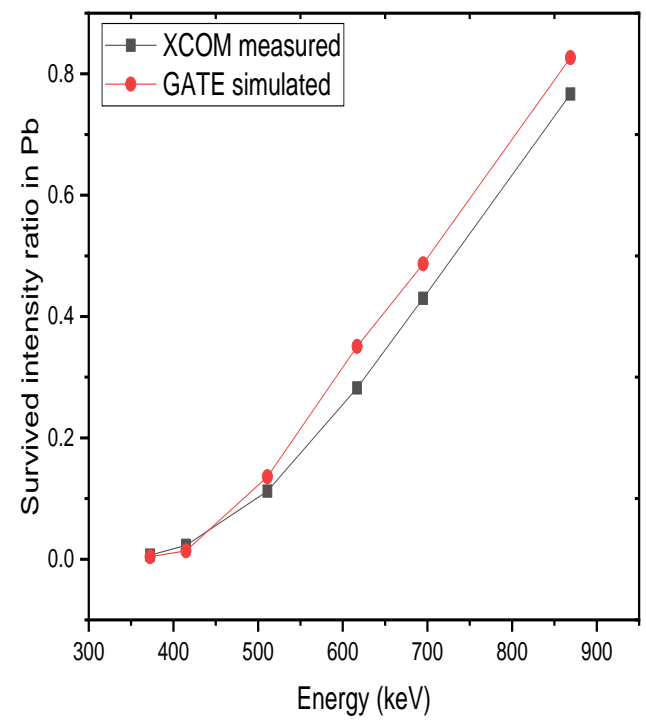

(A)

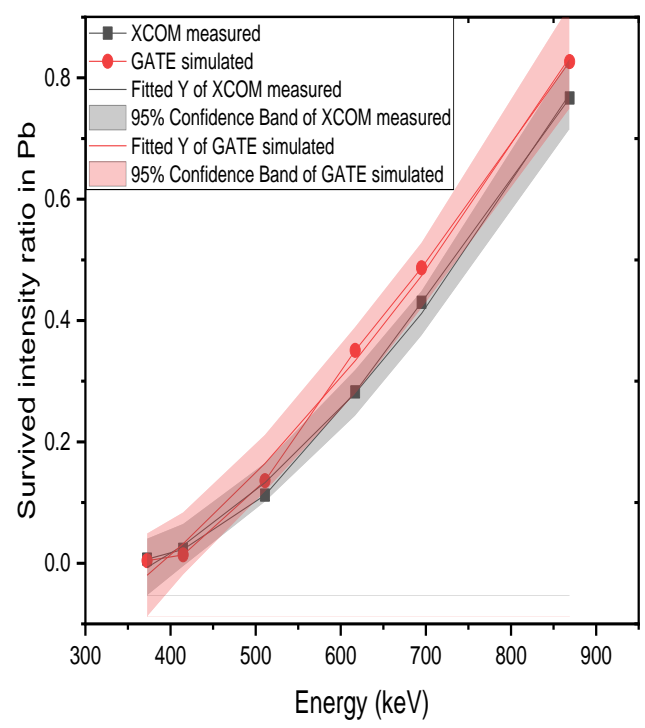

(B)

Figure 9: $\mathrm{XCOM}$ and GATE simulated data for survived intensity ratio in lead $(\mathrm{Pb})$ cuboid blocks (A) data points plot (B) polynomial-fitted data.

\section{Conclusion}

In this study, it has been shown that the simulation of a micro PET system employing pixelized dual layer GSO detector modules is feasible with GATE simulation platform. Subsequently, the developed GATE algorithm was used to evaluate the performance and response of the GSO detector by comparing the simulated data to the experimental and XCOM data to validate the simulation platform and procedure. The simulated data agreed well with the experimental and XCOM data. This agreement demonstrates the ability of GATE simulation platform in accurately modelling or simulating the performance and responses of the $\mathrm{Gd}_{2} \mathrm{SiO}_{5}$ (GSO) scintillator detector of the micro PET system. The developed and validated GATE simulation algorithms will be used in the future for simulating optimal collimator design, develop and assessing the image reconstruction algorithms and acquisition protocols for a novel medical imaging modality, which will be implemented in the existing micro PET prototype.

\section{Conflict of interest}

The authors declare that they have no competing interests.

\section{Acknowledgements}

The authors thank Prof. Chary Rangacharyulu from the University of Saskatchewan, Canada for allowing them (especially the first author) to work together on the "Novel medical imaging modality" project, under the Medical Imaging Group (International Collaboration). The first author also acknowledges the Tanzania Atomic Energy Commission (TAEC) for sponsoring his training at the University of Dar es Salaam. Lastly, the authors acknowledge the significant contributions of the late Prof. Peter K. Msaki, may his soul Rest in Eternal Peace.

\section{References}

Allison J, Amako K, Apostolakis J, Arce P, Asai M, Aso T, Bagli E, Bagulya A, Banerjee S, Barrand G, Beck BR, Bogdanov AG, Brandt D, Brown JMC, Burkhardt H, Canal P, ... Yoshida H 2016 
Nkuba et al. - A GATE-based Monte Carlo simulation of a dual-layer pixelized gadolinium ...

Recent developments in GEANT4 Nucl. Instruments Methods Phys. Res. Sect. A: Accel. Spectrometers, Detect. Assoc. Equip. 835: 186-225.

Brun R and Rademakers F 1997 ROOT-An object-oriented data analysis framework Nucl. Instruments Methods Phys. Res. Sect. A: Accel. Spectrometers, Detect. Assoc. Equip. 389: 81-86.

Buvat I and Castiglioni I 2002 Monte Carlo simulations in SPET and PET QJ Nucl. Med. 46(1): 48-61.

Fukuchi T, Okauchi T, Shigeta M, Yamamoto S, Watanabe Y and Enomoto S 2017 Positron emission tomography with additional $\gamma$-ray detectors for multipletracer imaging Med. Phys. 44(6): 2257-2266.

Jakoby BW, Bercier Y, Watson CC, Bendriem B and Townsend DW 2009 Performance characteristics of a new LSO PET/CT scanner with extended axial field-of-view and PSF reconstruction IEEE Trans. Nucl. Sci. 56(3): 633-639.

Jan S, Desbrée A, Pain F, Guez D, Comtat C, Gurden H, Kerhoas S, Lanièce P, Lefebvre F, Mastrippolito R and Trebossen R 2005 Monte Carlo simulation of the microPET FOCUS system for small rodents imaging applications IEEE Nucl. Sci. Symp. Conf. Rec. 3: 1653-1657.

Kawade K, Fukatsu K, Itow Y, Masuda K, Murakami T, Sako T, Suzuki K, Suzuki T and Taki K 2011 Study of radiation hardness of $\mathrm{Gd}_{2} \mathrm{SiO}_{5}$ scintillator for heavy ion beam J. Instrum. 6(9): T09004.

Khalil M 2017 Small animal micro-PET imaging: an overview. Egypt. J. Nucl. Med. 14(14): 8-27.

Lamare F, Turzo A, Bizais Y, Le Rest CC and Visvikis D 2006 Validation of a Monte Carlo simulation of the Philips Allegro/GEMINI PET systems using GATE. Phys. Med. Biol. 51(4): 943-962.

Lee WG, Lee DH, Kim YK, Kim JK and Park JW 2008 Growth and characteristics of $\mathrm{Gd}_{2} \mathrm{SiO}_{5}$ crystal doped $\mathrm{Ce}^{3+}$ J. Nucl. Sci. Technol. 45(5p): 572-574.

Lewellen TK 2008 Recent developments in
PET detector technology. Phys. Med. Biol. 53(17): R287-R317.

Matulewicz T, Korzecka K and Pytel Z 1996 Tests of GSO scintillator In: S. Zygmunt S and M. Popkiewicz M (Eds), Annual Reports: Nuclear Physics Division. 48-49.

Nikolopoulos D, Michail C, Valais I, Yannakopoulos P, Kottou S and Panayiotakis G 2014 GATE simulation of the biograph 2 PET/CT scanner. J. Nucl. Med. Radiat. Ther. 5(5): 201.

Okumura S, Yamamoto S, Watabe H, Kato N and Hamamura H 2015 Development of dual-layer GSO depth-of-interaction block detector using angled optical fiber Nucl. Instruments Methods Phys. Res. Sect. A: Accel. Spectrometers, Detect. Assoc. Equip. 781: 65-70.

Park SJ, Rogers WL, Huh S, Kagan H, Honscheid K, Burdette D, Chesi E, Lacasta C, Llosa G, Mikuz M, Studen A, Weilhammer P and Clinthorne NH 2007a A prototype of very high-resolution small animal PET scanner using silicon pad detectors Nucl. Instruments Methods Phys. Res. Sect. A: Accel. Spectrometers, Detect. Assoc. Equip. 570(3): 543-555.

Park SJ, Rogers WL and Clinthorne NH 2007b Design of a very high-resolution small animal PET scanner using a silicon scatter detector insert. Phys. Med. Biol. 52(15): 4653-4677.

Pietrzyk U 2017 GATE Users Guide V8.0 http://www.opengatecollaboration.org 8 : 134.

Qi Z, Shi C, Zhang G, Han Z and Hung HH 2003 Preparation and characterization of nanocrystalline $\mathrm{Gd}_{2} \mathrm{SiO}_{5}: \mathrm{Ce}$. Phys. Status Solidi Appl. Res. 195(2): 311-316.

Riehakainen L 2018 Performance evaluation of the Raycan Trans-PET/CT X5 all-digital small animal PET/CT scanner prototype. MSc Thesis, Åbo Akademi University.

Saaidi R, Toufique Y, Zeghari A, Kharrim A El, Cherkaoui R and Moursli E 2017 GATE Simulation of a clinical PET scanner: Influence of windows timing coincidences and dead time on count rate performance 
In: $4^{\text {th }}$ International Conference on Automation, Control Engineering and Computer Sciences (ACECS) Proceedings of Engineering and Technology-PET. 19: 31-35.

Santin G, Strul D, Lazaro D, Simon L, Krieguer M, Vieira Martins M, Breton V and Morel C 2003 GATE, a Geant4-based simulation platform for PET integrating movement and time management IEEE Nucl. Sci. Symp. Med. Imaging Conf. 50(5): 1516-1521.

Schmidtlein CR, Kirov AS, Nehmen SA, Erdi YE, Humm JL, Amols HI, Bidaut LM, Ganin A, Stearns CW, McDaniel DL and Hamacher KA 2006 Validation of GATE Monte Carlo simulations of the GE Advance/Discovery LS PET scanners. Med. Phys. 33(1): 198-208.

Tanaka M, Hara K, Kim S, Kondo K, Takano H, Kobayashi M, Ishibashi H, Kurashige K, Susa K and Ishii M 1998 Applications of cerium-doped gadolinium silicate $\mathrm{Gd}_{2} \mathrm{SiO}_{5}: \mathrm{Ce}$ scintillator to calorimeters in high-radiation environment. Nucl. Instruments Methods Phys. Res. Sect. A: Accel. Spectrometers, Detect. Assoc. Equip. 404(2-3): 283-294.

Toufique Y, El Moursli RC, Kaci M, El
Kharrim A and Merrouch R 2013 A benchmark of clinical PET using GATE simulation on the computing Grid In: Proceedings of IEEE/ACS International Conference on Computer Systems and Applications, AICCSA. 1-4.

Vandenbroucke A, Foudray AMK, Olcott PD and Levin CS 2010 Performance characterization of a new high-resolution PET scintillation detector. Phys. Med. Biol. 55(19): 5895-5911.

XCOM website: https://physics.nist.gov/PhysRefData/Xcom/ $\mathrm{html} /$ xcom1.html (Accessed on November 26-28, 2020).

Yamamoto S, Okumura S, Watabe T, Ikeda H, Kanai Y, Toshito T, Komori M, Ogata Y, Kato K and Hatazawa J 2015 Development of a prototype Open-close positron emission tomography system Rev. Sci. Instrum. 86(8): 084301.

Yamamoto S, Watabe H, Kato K and Hatazawa J 2012 Performance comparison of high quantum efficiency and normal quantum efficiency photomultiplier tubes and position sensitive photomultiplier tubes for high resolution PET and SPECT detectors. Med. Phys. 39(11): 6900-6907. 